\title{
An Early Morning Sputum Sample Is Necessary for the Diagnosis of Pulmonary Tuberculosis, Even with More Sensitive Techniques: A Prospective Cohort Study among Adolescent TB-Suspects in Uganda
}

\author{
Willy Ssengooba, ${ }^{1,2,3}$ David P. Kateete, ${ }^{1}$ Anne Wajja, ${ }^{2,3}$ Eric Bugumirwa, ${ }^{1,3}$ \\ Gerald Mboowa, ${ }^{1,3}$ Carolyn Namaganda, ${ }^{1,3}$ Germine Nakayita, ${ }^{1,3}$ Maria Nassolo,, 3 \\ Francis Mumbowa, ${ }^{1,3}$ Benon B. Asiimwe, ${ }^{1}$ James Waako, ${ }^{2,3}$ Suzanne Verver, ${ }^{4}$ \\ Philippa Musoke, ${ }^{2,3}$ Harriet Mayanja-Kizza, ${ }^{2,3}$ and Moses L. Joloba ${ }^{1,2,3}$ \\ ${ }^{1}$ Department of Medical Microbiology, School of Biomedical Sciences, Makerere University College of Health Sciences, \\ Kampala, Uganda \\ ${ }^{2}$ Infectious Diseases Institute, Makerere University College of Health Sciences, Kampala, Uganda \\ ${ }^{3}$ Iganga/Mayuge Demographic Surveillance Sites, School of Public Health, Makerere University College of Health Sciences, \\ Kampala, Uganda \\ ${ }^{4}$ KNCV Tuberculosis Foundation, The Hague and CINIMA, Academic Medical Centre, Parkstraat 17, \\ 2514 JD The Hague, The Netherlands
}

Correspondence should be addressed to Willy Ssengooba, willyssengooba@gmail.com and Moses L. Joloba, moses.joloba@case.edu

Received 17 October 2012; Revised 19 November 2012; Accepted 20 November 2012

Academic Editor: Carlo Garzelli

Copyright ( $(2012$ Willy Ssengooba et al. This is an open access article distributed under the Creative Commons Attribution License, which permits unrestricted use, distribution, and reproduction in any medium, provided the original work is properly cited.

The World Health Organization (WHO) recommends collection of two sputum samples for tuberculosis (TB) diagnosis, with at least one being an early morning (EM) using smear microscopy. It remains unclear whether this is necessary even when sputum culture is employed. Here, we determined the diagnostic yield from spot and the incremental yield from the EM sputum sample cultures among TB-suspected adolescents from rural Uganda. Sputum samples (both spot and early-morning) from 1862 adolescents were cultured by the Lowenstein-Jensen (LJ) and Mycobacterium Growth Indicator Tube (MGIT) methods. For spot samples, the diagnostic yields for TB were $19.0 \%$ and 57.1\% with LJ and MGIT, respectively, whereas the incremental yields (not totals) of the early-morning sample were $9.5 \%$ and $42.9 \%(P<0.001)$ with LJ and MGIT, respectively. Among TB-suspected adolescents in rural Uganda, the EM sputum culture has a high incremental diagnostic yield. Therefore, EM sputum in addition to spot sample culture is necessary for improved TB case detection.

\section{Background}

Tuberculosis (TB) remains a global emergency, causing high mortality and morbidity particularly in sub-Saharan Africa [1]. Some studies have shown that the incidence of TB in adolescents (12-18 years old) has increased by $22 \%$ compared with a $38 \%$ decrease in children less than 5 years old [2].
Although there is scant data on TB in adolescents in Uganda and worldwide in general, their protective response against Mycobacterium tuberculosis (MTB) infection seems to be less effective [3]. Adolescents also have unique clinical presentations for TB; they are more asymptomatic and are more likely to have cavitary disease [4]. Reports indicate that many adolescents with active TB are diagnosed during late stage of the disease [2]. Additionally, the demographic and 
clinical characteristics of adolescents with TB differ from adults and children [5]. Furthermore, it is quite difficult to obtain quality sputum samples, which makes TB diagnosis among adolescents challenging. Indeed, many adolescents with $\mathrm{TB}$ are prone to producing smear negative sputum samples [6].

The World Health organization (WHO) and the International Union Against Tuberculosis and Lung Diseases (IUATLD) recommended collection of two sputum samples for smear microscopy with at least one being an earlymorning (EM) sample. This aimed at reducing the workload per serial sample examined $[7,8]$. However, these recommendations seem to work in settings serving the general population where external quality assurance (EQA) methods are well established. As such, those recommendations may not address the number of samples required in other situations where sputum culture is indicated.

The definitive diagnosis of TB depends mainly on culture of MTB from the clinical samples [9]. According to the mathematical models by Dowdy et al., expanded use of TB culture may have an immediate impact on TB burden in that it can reduce TB rates in high burdened countries [10]. However, this concept remains less applied in resource limited countries that are most burdened by TB. This is largely due to the high expenses and cost of infrastructure required [11]. For instance, there is only one public TB culture facility in Uganda, located in Kampala, the capital.

Despite the move to expand and improve laboratory capacity to perform TB culture [12], and the availability of automated liquid culture systems which requires less labor [13], little has been done to assess the impact and cost effectiveness including operational studies for performing $\mathrm{TB}$ culture in resource limited countries. The situation is even worse when adolescents are involved in that culture would be the most appropriate for diagnosis [14].

Whereas the number and type of sputum samples required for smear microscopy have been standardized, the number and type of sputum samples required for culture have not been well standardized. Furthermore, while there is a significant diagnostic gain from EM sputum samples when using smear microscopy, it is not clear whether this gain remains significant when using more sensitive techniques such as culture particularly among adolescents. This study therefore aimed at determining the diagnostic yield of the spot and the incremental yield of EM sputum sample cultures for diagnosis of $\mathrm{TB}$ among adolescents in rural Uganda.

\section{Methods}

2.1. Study Design and Setting. This study was a subcomponent of a large observational study undertaken by Makerere University School of Public Health, which enrolled 5000 adolescents in rural Uganda, in preparation for TB vaccine trials. The study was done in a Demographic Surveillance Site (DSS) located in Iganga and Mayuge districts in Eastern Uganda ( $\sim 150 \mathrm{~km}$ from Kampala, the capital).

The adolescent TB cohort (12-18 years of age) enrolled 5,000 participants from both primary and secondary schools within the DSS. It enrolled participants with a positive Tuberculin Skin Test (TST) and at least one TB related symptom or history of contact with a TB diseased person. Participants who had plans of relocating within the study period or those medically unfit for collection of sputum were excluded. Clinical evaluation for TB disease included giving two sputum samples; one collected on spot under supervision (spot sample) on day one and another on day 2 by the participant without supervision (EM sample).

Sputum samples were obtained daily from the participants by nurses and transported in cold boxes (temperature $\leq 8^{\circ} \mathrm{C}$ ) to a reference mycobacteriology laboratory (biosafety level 3) where culture and analysis were done. This laboratory is located in the Department of Medical Microbiology, Makerere University College of Health Sciences Kampala. Samples collected after midday were refrigerated till the next day.

Thus, the study analysis included study participants with both EM and spot sputum samples submitted for culture.

2.1.1. Laboratory Procedures. Sputum samples were processed according to standard procedures[15] and simultaneously inoculated in Lowenstein Jensen (LJ) (Becton and Dickson, Franklin Lakes, NJ, USA) and Mycobacterium Growth Indicator Tube (MGIT) (Becton and Dickson, Franklin Lakes, NJ, USA) culture bottles with growth media as described elsewhere $[15,16]$. Smears for fluorescent microscopy were also done according to standard procedures [15].

For purity, MGIT-positive cultures were further subcultured at $37^{\circ} \mathrm{C}$ on blood agar for 24 hours and Ziehl Neelsen (ZN) smears done. Cultures which were ZN-positive and pure (i.e., no growth on blood agar) were subjected to Capilia TB Neo (TAUN, Numazu, Japan) for identification of the MTBC [17]. Cultures with growth on blood agar but also ZN-positive were sub-cultured again as described above; the persistent $\mathrm{ZN}$-negative cultures with growth on blood agar were considered contaminated. All ZN-positive, blood agar negative, and Capilia TB Neo negative samples were regarded Mycobacteria Other Than Tuberculosis (MOTT); these were subjected to the Genotype AS assay (Hain Lifescience, Nehren, Germany) for speciation. Otherwise absence of colonies on LJ or fluorescence in MGIT was considered negative. The data were entered into a computerized laboratory access database linked with patient records on sample reporting form.

2.1.2. Data Analysis. The primary outcome of our analysis was culture positive TB, defined as MTBC positive upon LJ or MGIT culture from either spot or EM samples. Laboratory data were analyzed for the diagnostic yield; this referred to the number of TB cases detected by each sputum sample type (spot or EM) irrespective of whether the comparator was positive. The data were analyzed for the Incremental diagnostic yield; this referred to the yield of culture positive EM samples in terms of TB cases detected when the spot samples from the same case was negative. Contamination rate was calculated as the total number of cultures per sample 
TABLE 1: Characteristics of the six patients with MTBC-positive smears and the 21 patients with MTBC-positive culture sputum sample type among adolescent TB suspects in rural Uganda $(n=1862)$.

\begin{tabular}{|c|c|c|c|c|c|}
\hline \multirow[b]{2}{*}{ Characteristics } & \multicolumn{2}{|c|}{ Smear positive* } & \multicolumn{2}{|c|}{ Culture positive (MTBC)* } & \multirow{2}{*}{$\begin{array}{l}\text { Total enrolled } \\
n(\%) \text { of Tota }\end{array}$} \\
\hline & $\begin{array}{c}\text { Spot } \\
n(\%) \text { of Total }\end{array}$ & $\begin{array}{c}\text { EM } \\
n(\%) \text { of Total }\end{array}$ & $\begin{array}{c}\text { Spot } \\
n(\%) \text { of Total }\end{array}$ & $\begin{array}{c}\text { EM } \\
n(\%) \text { of Total }\end{array}$ & \\
\hline Total & 3 & 6 & 9 & 15 & 1862 \\
\hline \multicolumn{6}{|l|}{ Sex } \\
\hline Male & $1(33.3)$ & $4(66.7)$ & $4(44.4)$ & $7(46.7)$ & $1069(57.4)$ \\
\hline Female & $2(66.7)$ & $2(33.3)$ & $5(55.6)$ & $8(53.3)$ & $793(42.6)$ \\
\hline \multicolumn{6}{|l|}{ Age ranges } \\
\hline $12-14$ & $0(0.0)$ & $1(16.7)$ & $5(55.6)$ & $1(6.7)$ & $805(43.2)$ \\
\hline $15-16$ & $3(100)$ & $4(66.7)$ & $4(44.4)$ & $8(53.3)$ & $543(29.1)$ \\
\hline $17-18$ & $0(0.0)$ & $1(16.7)$ & $0(0.0)$ & $7(46.7)$ & $514(27.6)$ \\
\hline \multicolumn{6}{|l|}{ Volume } \\
\hline$\leq 5 \mathrm{~mL}$ & $1(33.3)$ & $3(50.0)$ & $3(33.3)$ & $9(60.0)$ & $1226(65.8)$ \\
\hline$>5 \mathrm{~mL}$ & $2(66.7)$ & $3(50.0)$ & $6(66.7)$ & $6(40.0)$ & $636(34.2)$ \\
\hline \multicolumn{6}{|l|}{ Consistency } \\
\hline Salivary & $2(66.7)$ & $3(50.0)$ & $4(44.4)$ & $7(46.7)$ & $872(46.8)$ \\
\hline Mucosalivary & $1(33.3)$ & $3(50.0)$ & $3(33.3)$ & $2(13.3)$ & $350(18.8)$ \\
\hline Mucoid & $0(0.0)$ & $0(0.0)$ & $1(11.1)$ & $2(13.3)$ & $360(19.3)$ \\
\hline Mucopurulent & $0(0.0)$ & $0(0.0)$ & $1(11.1)$ & $3(20.0)$ & $213(11.4)$ \\
\hline Purulent & $0(0.0)$ & $0(0.0)$ & $0(0.0)$ & $1(6.7)$ & $67(3.6)$ \\
\hline
\end{tabular}

EM: Early morning, MTBC: mycobacterium tuberculosis complex, $n$ : number, \%: percentage; ${ }^{*}$ The 3 smear positive on spot were also detected by the early morning sample, also 3 participants were positive on both spot and early morning sputum cultures.

contaminated over the total number of cultures per sample type inoculated.

Statistical analysis was performed with Stata SE software version 11 (Stata Corp LP, College station TX, USA). The unit of analysis was the sample type to show the diagnostic yield from each and Incremental yield for EM sputum samples collected for culture. A $P$-value of $<0.05$ was considered statistically significant.

2.1.3. Ethical Considerations. This study operated under a waiver of consent since it involved secondary data analysis from the major study that previously obtained ethical approval from the Makerere University College of Health Sciences School of Public Heath Institutional Review Board (IRB) and the Uganda National Council for Science and Technology (UNCST).

\section{Results}

A total of 5000 adolescent participants were screened for TB, of whom $2418(48.4 \%)$ met the study criteria for a TB suspect. Of the $2418 \mathrm{~TB}$ suspects, $803(33.2 \%)$ had a positive TST, $237(57.0 \%)$ had contact with a TB patient, and $1378(57.0 \%)$ had at least one TB symptom. Furthermore, $556(22.9 \%)$ were excluded due to challenges with sample collection; $50(2.1 \%)$ had dry cough hence did not provide sputum, 301 (12.4\%) failed to deliver an EM sample, 110 (4.5\%) relocated prior to sputum collection, while $95(3.9 \%)$ missed the study visit to the collection point. Thus, the

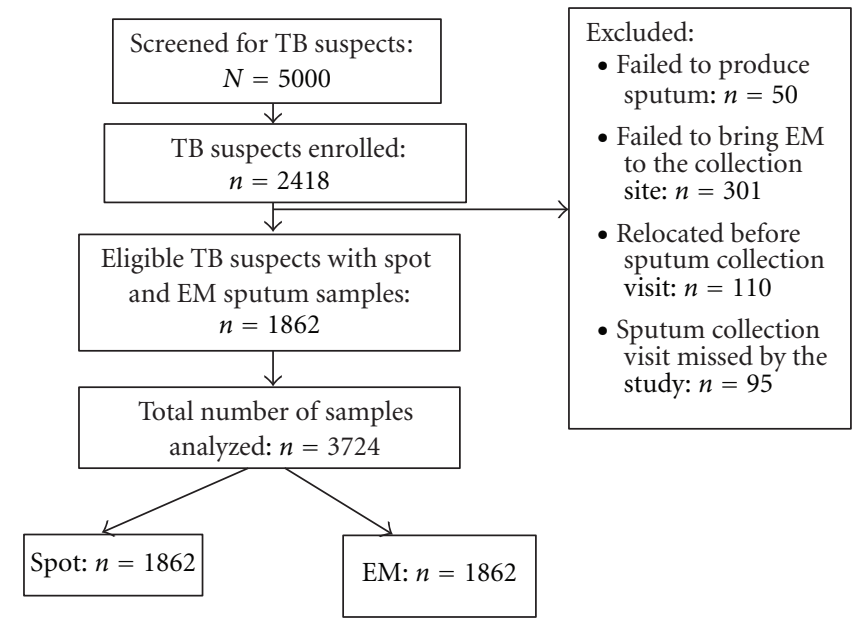

Figure 1: Study flow chart for 1862 adolescent TB suspects based on spot and EM sputum samples.

analysis was limited to 1862 eligible participants who had both the EM and spot samples, Figure 1.

Of the 1862 participants, six $(0.3 \%)$ were MTBC-positive based on smear microscopy while $21(1.1 \%)$ were MTBCpositive based on culture (confirmed by Capilia Neo TB assay as described in methods) hence they were regarded as true TB cases (Table 1).

3.1. Mycobacterial Yield of Spot and Early Morning Sputum Samples. Based on LJ culture, only six $(0.4 \%)$ samples were 
TABLE 2: Mycobacterial yield from EM and spot sputum samples by different culture methods $(n=1862)$.

\begin{tabular}{|c|c|c|c|c|c|}
\hline Culture method & Results & $\begin{array}{c}\text { Spot samples } \\
n(\%)\end{array}$ & $\begin{array}{c}\text { EM samples } \\
n(\%)\end{array}$ & $\begin{array}{c}\text { EM and spot samples } \\
n(\%)\end{array}$ & $\begin{array}{l}\text { Total } \\
n(\%)\end{array}$ \\
\hline \multirow{4}{*}{$\mathrm{LJ}(P<0.001)$} & MTBC positive & $1(0.1)$ & $2(0.1)$ & $3(0.2)$ & $6(0.3)$ \\
\hline & MOTT & $0(0.0)$ & $0(0.0)$ & $0(0.0)$ & $0(0.0)$ \\
\hline & Culture negative & $1831(98.3)$ & $1806(97.0)$ & $1859(99.8)$ & $1772(95.2)$ \\
\hline & Contaminated & $30(1.61)$ & $54(2.9)$ & $0(0.0)$ & $84(4.5)$ \\
\hline \multirow{4}{*}{$\operatorname{MGIT}(P<0.001)$} & MTBC positive & $6(0.3)$ & $12(0.6)$ & $3(0.2)$ & $21(1.1)$ \\
\hline & MOTT & $106(5.7)$ & $119(6.4)$ & $0(0.0)$ & $225(12.1)$ \\
\hline & Culture negative & $380(20.4)$ & $301(16.2)$ & $1859(99.8)$ & $681(36.6)$ \\
\hline & Contaminated & $436(23.4)$ & $499(26.8)$ & $0(0.0)$ & $935(50.2)$ \\
\hline
\end{tabular}

EM: early morning; MTBC: mycobacterium tuberculosis complex, MOTT: mycobacterium other than tuberculosis, LJ: Lowenstein Jensen, MGIT: mycobacterium growth indicator tube. IY: incremental yield, LJ: Lowenstein Jensen, MGIT: mycobacterium growth indicator tube.

TABLE 3: Diagnostic yield of TB for the spot and incremental diagnostic yield (not total) of EM sputum sample cultures $(n=21)$.

\begin{tabular}{lccc}
\hline Culture method & Yield of spot & Incremental diagnostic yield for EM (observed) & Total number of TB cases detected \\
$n(\%)$ & $(\%)$ & $6(28.6)$ \\
\hline LJ & $4(19.0)$ & $2(9.5)$ & $21(100)$ \\
MGIT & $12(57.1)$ & $9(42.9)$ & \\
\hline
\end{tabular}

$n$ : number, IY: incremental yield, LJ: Lowenstein Jensen, MGIT: mycobacterium growth indicator tube, EM: early morning.

MTBC-positive meaning the TB case detection based on LJpositive culture were six cases: these were also positive by smear microscopy. Of the six LJ-positive cases, one $(0.1 \%)$ was from the spot sample, two $(0.2 \%)$ from EM, $(P<0.001)$ while three $(0.5 \%)$ were from both spot and EM samples. The LJ contamination rate for spot samples was $1.6 \%$ while it was $2.9 \%$ for the EM samples $(P=0.008)$.

On the other hand, MGIT culture detected 21 TB cases (including the six LJ-positive ones); six (0.3\%) on spot, EM $12(0.6 \%)$, and three $(0.2 \%)$ on both spot and EM samples $(P<0.001)$. Furthermore, with MGIT, 225 MOTT $(12.1 \%)$ were also detected of which $106(5.7 \%)$ and $119(6.4 \%)$ $(P<0.001)$ were from spot and EM samples, respectively, (Table 2).

The MOTT were speciated as follows: M. fortuitum 143 (63.6\%); M. Szugai 32 (14.2\%); M. gordonae 21 (9.3\%); $M$. intracellulare 11 (4.9\%); M. scrofulaceum 7 (3.1\%); M. lentiflavum 7 (3.1\%); M. peregrinum 4 (1.8\%).

Overall, 436/1862 (23.4\%) of the spot and $499 / 1862$ (26.8\%) $(P<0.001)$ of the EM sample cultures were contaminated (Table 2). The most common contaminants were Gram positive cocci.

3.2. Diagnostic Yield of TB for the Spot and the Incremental Yield of the Early Morning Sputum Sample Cultures. The TB diagnostic yield of the spot sample was $4(19.0 \%)$ and $12(57.1 \%)$ on LJ and MGIT, respectively. The incremental diagnostic yield (not total) of the EM sample was $2(9.5 \%)$ and $12(42.9 \%)(P<0.001)$ on LJ and MGIT, respectively (Table 3).

\section{Discussion}

This study has revealed that among adolescent TB suspects in rural Uganda, an EM sputum sample culture has a higher incremental diagnostic yield. However, the study also found that EM culture also detects more MOTT in comparison with the spot sample. The diagnostic gain of the EM sputum culture given a negative spot sputum sample culture was 9.5\% on LJ culture and $42.9 \%$ on MGIT.

Given the sensitivity of sputum culture especially with liquid systems (MGIT) over smear microscopy, one would imagine the incremental diagnostic yield of the EM sputum sample to be negligible; however, this is contrary to our findings which are in agreement with the previous studies by either microscopy or culture [7, 18-20]. Findings in this study further agree with previous studies which reported a significant gain from the EM sample [21-23]. This may be due in part to the accumulation of sputum in the lungs overnight, resulting in a concentration of bacilli in the EM samples. In contrast, patients may be more active during the day and may shed bacilli intermittently, thus reducing the yield of bacilli in spot sputum samples. These facts further support guidelines to examine EM samples in addition to spot sputum samples before declaring a TB suspect as negative for pulmonary TB.

Solid culture detected less TB cases and it was less contaminated compared to MGIT and this difference, in terms of sample type, was in agreement with previous studies [21, $24,25]$. However, Tortoli et al. documented the opposite with LJ culture [26]. The low yield of LJ in comparison with MGIT is unlikely to be attributed to decontamination procedures as samples were processed similarly using the same procedures and with the same homogenized sputum samples inoculated in both culture media. The lower contamination rate could be due to the fact that we used commercially prepared LJ which was properly quality-controlled and was less likely to have attracted contaminants. 
The high contamination rate with MGIT could partly be due to the fact that this method uses highly nutritious medium that easily supports growth of other bacteria. Alternatively, the long distance from the sputum sample collection site ( 3 hrs drive) to the laboratory could have contributed in that it facilitated rapid multiplication of normal flora, which are mainly Gram positive cocci [26]. We observed less contamination with spot samples (which were supervised at collection sites) when compared with the EM samples (which were not unsupervised at collection sites); however, this may not be significant since we did not provide patients with water or other materials to minimize normal flora prior to expectoration. Nevertheless, this finding is supported by similar findings in a previous study [27]. It is also worth noting that high contamination rates may have contributed to underestimation of TB cases by MGIT and increased the cost of doing the test. Furthermore, the increased yield of the second sputum culture could be partly due to the EM samples detecting those which were contaminated on spot; however contamination is unlikely to explain the increased yield of EM culture since a similar scenario of increased yield was also documented by previous studies with low contamination rates [21,22].

In agreement with previous studies [28], we found that LJ may have an unfavorable environment to support MOTT growth since all the isolated mycobacteria were MTBC [28] and a significant number of MOTT were isolated with MGIT. This is mainly due to the fact that MGIT is more sensitive and facilitates rapid multiplication of fast growing MOTT than LJ. That is why WHO emphasizes rapid identification of any MGIT which is ZN positive to be identified before reporting the results [29]. However this is contrary with another study which documented almost similar number of MOTT isolated on LJ and MGIT cultures [26]. This may be attributed to regional differences in distribution of MOTT as most MOTT species from that study are different from those identified in ours.

Since most mycobacteria are similar phenotypically, the prevalence of MOTT in this study population is worrying in that they may distort the immunogenicity data in the planned TB vaccine trials. Furthermore, much as we did not document any disease due to MOTT infection, studies on MOTT are needed since diseases due to these organisms have been documented in other settings [30]. Additionally, as the number of participants who failed to return with an EM sample in this study was high 301/2418 (12.4\%), future studies considering other sputum sample collection strategies, such as, the "Front Loading" strategy would also be important.

Our study had some limitations; first, we did not separate mycobacterial yield of spot and EM sputum samples collected at baseline and follow-up; therefore, differences in sample composition may have occurred at different time points during the study. Furthermore, the main study documented few laboratory confirmed TB cases. This may be different when other forms of TB diagnosis including clinical diagnosis are considered. However, given a bigger sample size, our findings provide significant information for future studies on TB among adolescents.
The samples analyzed were collected in a research setting with supervision of the spot sample collection as well as being analyzed in a purely research BSL-3 TB culture facility; hence, much as our findings have implications for controlled research settings like clinical trials, they may not represent what happens in routine practice.

Additionally, we identified MTBC with Capilia TB Neo, which may give false negatives as documented by previous studies [30, 31]; however, all MOTT and MTBC were reconfirmed using the Line Probe Hain CM and AS genotype assays.

In conclusion, early morning sputum culture has a high incremental diagnostic yield for TB among adolescents in Uganda. Among TB suspected adolescents, early morning sputum in addition to spot sample culture is necessary for better TB case detection. MGIT system cultivated all the MTBC cases as well as MOTT and majority were from early morning sputum samples.

\section{Acknowledgments}

The authors are thankful to the participants from Iganga/ Mayuge Iganga/Mayuge Demographic Surveillance Sites (DSS) and to the technical team of Mycobacteriology (BSL-3) Laboratory of Makerere University College of Health Sciences. This study was funded by the European and Developing Countries Clinical Trials Partnership (EDCTP) with administrative support from Infectious Diseases Institute (IDI) and Iganga/Mayuge DSS of Makerere University College of Health Sciences.

\section{References}

[1] World Health Organization, "Global tuberculosis control report, 2011," http://www.who.int/tb/publications/global_ report/2011/gtbr11_full.pdf.

[2] C. Didilescu, E. Ibraim, and M. Tigau, "The epidemiological profile and current evolutionary trends in tuberculosis in adolescents (15-19 years old) in the capital," Pneumoftiziologia, vol. 46, no. 3, pp. 193-199, 1997.

[3] R. L. Nemir, "Perspectives in adolescent tuberculosis: three decades of experience," Pediatrics, vol. 78, no. 3, pp. 399-405, 1986.

[4] A. Kam, L. Ford-Jones, P. Malloy, K. Khan, and I. Kitai, “Active tuberculosis among adolescents in Toronto, Canada: clinical features and delays in diagnosis," Pediatric Infectious Disease Journal, vol. 26, no. 4, pp. 355-356, 2007.

[5] L. De Pontual, L. Balu, P. Ovetchkine et al., "Tuberculosis in adolescents: a French retrospective study of 52 cases," Pediatric Infectious Disease Journal, vol. 25, no. 10, pp. 930-932, 2006.

[6] Y. L. J. Byeon, L. J. Chul, Y. Young et al., "Three cases of pulmonary and/or intestinal tuberculosis in adolescents," Korean Journal of Pediatric, vol. 50, no. 11, pp. 1134-1138, 2007.

[7] 2006, WHO: tuberculosis Coalition for Technical Assistance. International Standards for Tuberculosis Care (ISTC). The Hague: tuberculosis Technical Assistance, http://www.who .int/tb/publications/2006/istc_report.pdf.

[8] A. D. Harries, N. B. Mphasa, C. Mundy, A. Banerjee, J. H. Kwanjana, and F. M. L. Salaniponi, "Screening tuberculosis suspects using two sputum smears," International Journal of Tuberculosis and Lung Disease, vol. 4, no. 1, pp. 36-40, 2000. 
[9] H. Getahun, M. Harrington, R. O’Brien, and P. Nunn, "Diagnosis of smear-negative pulmonary tuberculosis in people with HIV infection or AIDS in resource-constrained settings: informing urgent policy changes," The Lancet, vol. 369, no. 9578, pp. 2042-2049, 2007.

[10] D. W. Dowdy, R. E. Chaisson, G. Maartens, E. L. Corbett, and S. E. Dorman, "Impact of enhanced tuberculosis diagnosis in South Africa: a mathematical model of expanded culture and drug susceptibility testing," Proceedings of the National Academy of Sciences of the United States of America, vol. 105, no. 32, pp. 11293-11298, 2008.

[11] L. Apers, J. Mutsvangwa, J. Magwenzi et al., "A comparison of direct microscopy, the concentration method and the Mycobacteria Growth Indicator Tube for the examination of sputum for acid-fast bacilli," International Journal of Tuberculosis and Lung Disease, vol. 7, no. 4, pp. 376-381, 2003.

[12] 2006, WHO: improving the diagnosis and treatment of smear-negative pulmonary and extra- pulmonary tuberculosis among adults and adolescents. Recommendations for HIVprevalent and resource-constrained settings, http://www.who .int/tb/publications/2006/tbhiv_recommendations.pdf.

[13] G. L. Woods, "The mycobacteriology laboratory and new diagnostic techniques," Infectious Disease Clinics of North America, vol. 16, no. 1, pp. 127-144, 2002.

[14] A. M. Mandalakas and J. R. Starke, "Current concepts of childhood tuberculosis," Seminars in Pediatric Infectious Diseases, vol. 16, no. 2, pp. 93-104, 2005.

[15] B. E. Strong and G. P. Kubica, "Isolation and identification of Mycobacterium tuberculosis: a guide for the level II laboratory," Centers for Disease Control; HHS Publication No. (CDC) 818390, http://books.google.co.ug/books?id=_VurMQEACAAJ, 1981.

[16] S. H. Siddiqi, 2006, MGIT Procedure Manual, For BACTEC MGIT 960 TB System, Specially Prepared for FIND MGIT demonstration Project, http://www.finddiagnostics.org/export/sites/default/resource-centre/find_documentation/ pdfs/mgit_manual_nov_2007.pdf.

[17] K. Hirano, A. Aono, M. Takahashi, and C. Abe, "Mutation including IS6110 insertion in the gene encoding the MPB64 protein of Capilia TB- negative Mycobacterium tuberculosis isolates," Journal of Clinical Microbiology, vol. 42, no. 1, pp. 390-392, 2004.

[18] WHO Strategic and Technical Advisory Group for Tuberculosis, "Report on conclusions and recommendations," June 2007, http://www.who.int/tb/events/stag_report_2007.pdf .

[19] S. Rohit, S. Mukerjee, S. Neeta, and P. P. Sharma, "Diagnosis of tuberculosis under RNTCP: examination of two or three sputum specimens," Indian Journal of Tuberculosis, vol. 48, no. 13, 16 pages, 2001.

[20] O. D. Schoch, P. Rieder, C. Tueller et al., "Diagnostic yield of sputum, induced sputum, and bronchoscopy after radiologic tuberculosis screening," American Journal Respiratory Critical Care Medicine, vol. 175, pp. 80-86, 2007.

[21] P. Monkongdee, K. D. McCarthy, K. P. Cain et al., "Yield of acid-fast smear and mycobacterial culture for tuberculosis diagnosis in people with human immunodeficiency virus," American Journal of Respiratory and Critical Care Medicine, vol. 180, no. 9, pp. 903-908, 2009.

[22] D. A. J. Moore, C. A. W. Evans, R. H. Gilman et al., "Microscopic-observation drug-susceptibility assay for the diagnosis of TB," The New England Journal of Medicine, vol. 355, no. 15, pp. 1539-1550, 2006.

[23] W. Ssengooba, N. Kiwanuka, D. P. Kateete et al., "Incremental yield of serial sputum cultures for diagnosis of tuberculosis among HIV infected smear negative pulmonary TB suspects in Kampala, Uganda," PLoS ONE, vol. 7, Article ID e37650, 5 pages, 2012.

[24] V. N. Chihota, A. D. Grant, K. Fielding et al., "Liquid vs. solid culture for tuberculosis: performance and cost in a resourceconstrained setting," International Journal of Tuberculosis and Lung Disease, vol. 14, no. 8, pp. 1024-1033, 2010.

[25] J. J. Lee, J. Suo, C. B. Lin, J. D. Wang, T. Y. Lin, and Y. C. Tsai, "Comparative evaluation of the BACTEC MGIT 960 system with solid medium for isolation of mycobacteria," International Journal of Tuberculosis and Lung Disease, vol. 7, no. 6, pp. 569-574, 2003.

[26] E. Tortoli, P. Cichero, C. Piersimoni, M. T. Simonetti, G. Gesu, and D. Nista, "Use of BACTEC MGIT 960 for recovery of mycobacteria from clinical specimens: multicenter study," Journal of Clinical Microbiology, vol. 37, no. 11, pp. 3578-3582, 1999.

[27] E. L. N. Maciel, T. N. do Prado, R. L. Peres, M. Palaci, J. L. Johnson, and R. Dietze, "Guided sputum sample collection and culture contamination rates in the diagnosis of pulmonary TB," Jornal Brasileiro de Pneumologia, vol. 35, no. 5, pp. 460-463, 2009.

[28] W. Worodria, J. Anderson, A. Cattamanchi et al., "The role of speciation in positive Lowenstein-Jensen culture isolates from a high tuberculosis Burden country," PLOS ONE, vol. 11, Article ID e27017, 6 pages, 2011.

[29] World Health Organization, TB diagnostics and laboratory strengthening-WHO policy, 2007, http://www.who.int/tb/ policy_liquid_medium_for_culture_dst/en/index.html.

[30] K. D. McCarthy, K. P. Cain, K. L. Winthrop et al., "Nontuberculous mycobacterial disease in patients with HIV in Southeast Asia," American Journal Respiratory Critical Care Medicine, vol. 185, no. 9, pp. 981-988, 2012.

[31] C. Muchwa, J. Akol, A. Etwom et al., "Evaluation of Capilia TB assay for rapid identification of Mycobacterium tuberculosis complex in BACTEC MGIT 960 and BACTEC, 9120 blood cultures," BMC Research Notes, article 44, vol. 5, no. 1, 2012. 


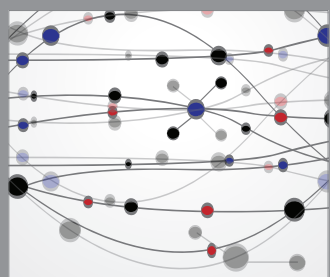

The Scientific World Journal
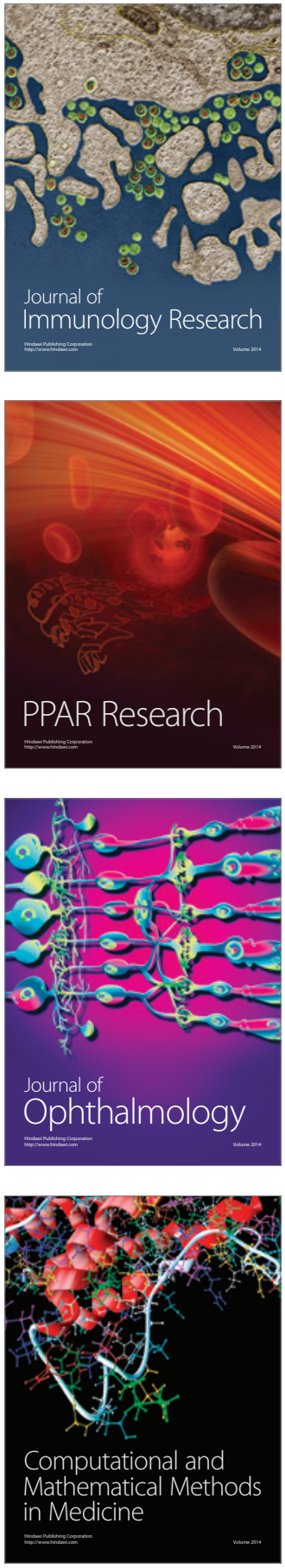

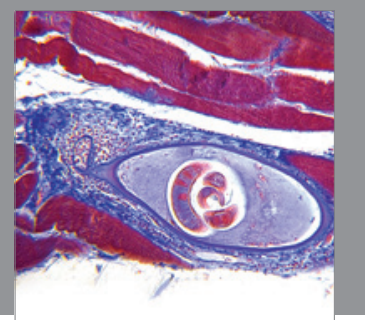

Gastroenterology

Research and Practice
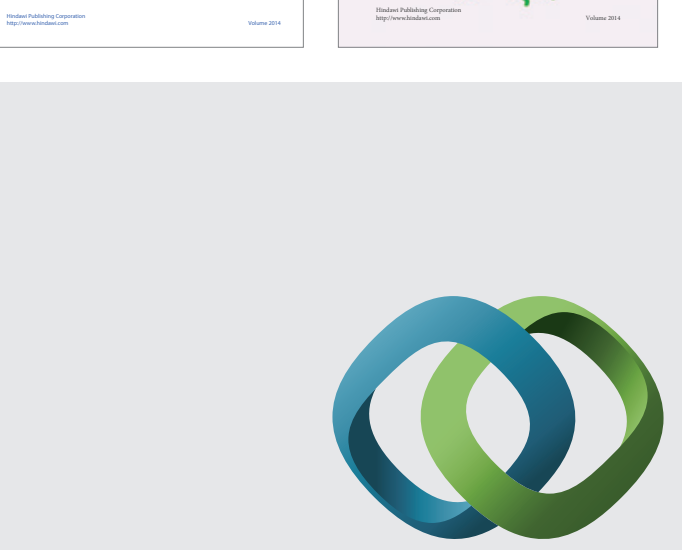

\section{Hindawi}

Submit your manuscripts at

http://www.hindawi.com
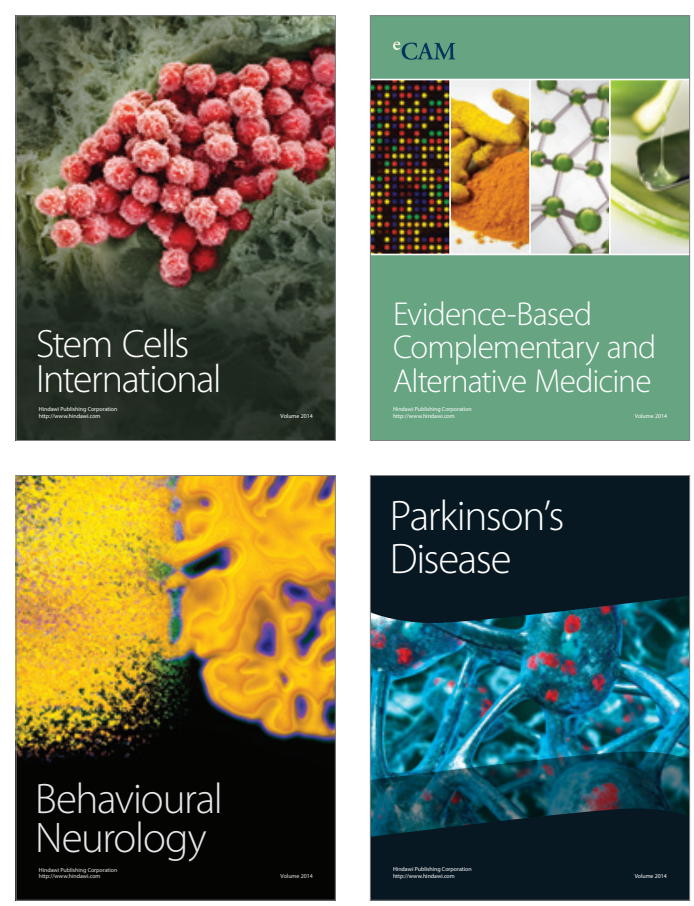

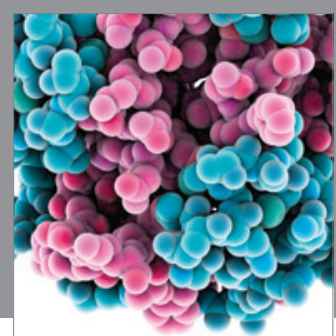

Journal of
Diabetes Research

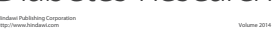

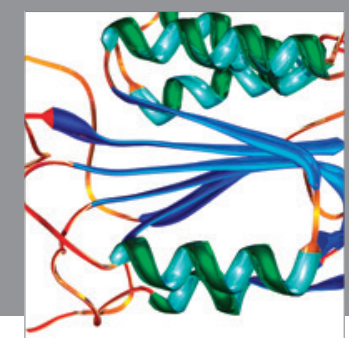

Disease Markers
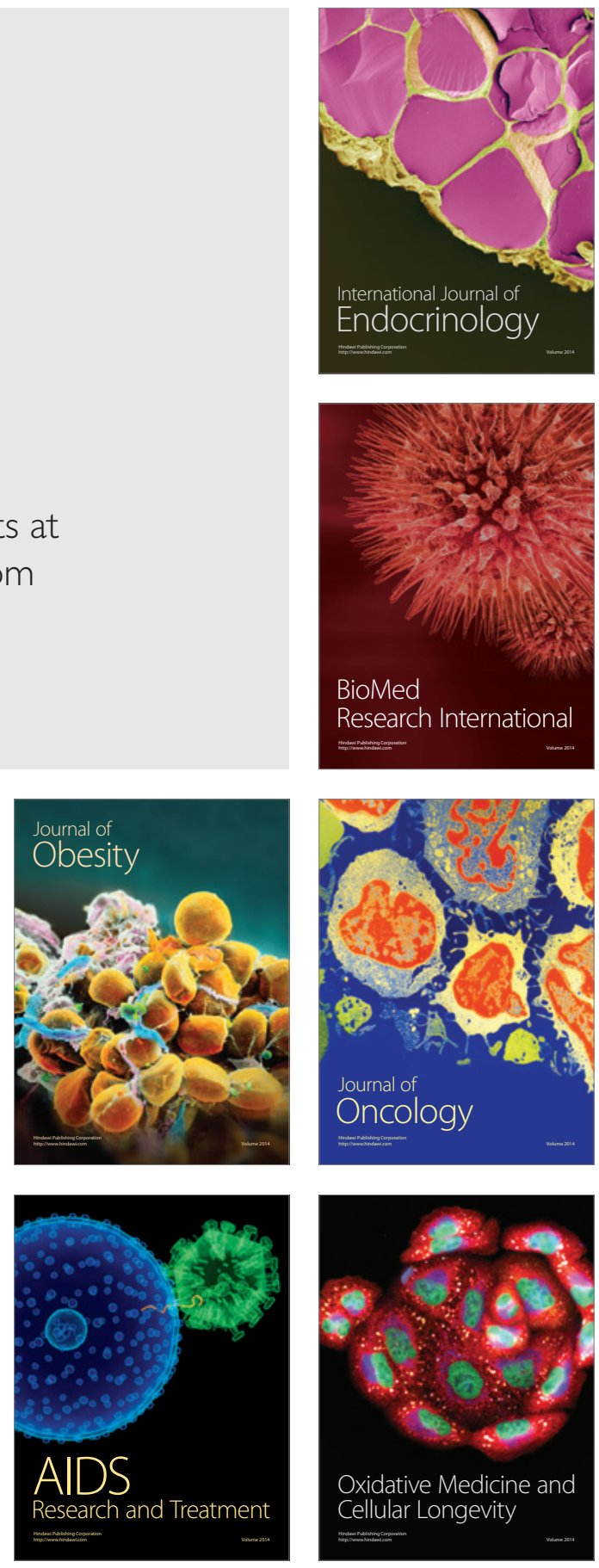